NISTIR 8397

\title{
Guidelines on Minimum Standards for Developer Verification of Software
}

\author{
Paul E. Black \\ Barbara Guttman \\ Vadim Okun
}

This publication is available free of charge from:

https://doi.org/10.6028/NIST.IR.8397

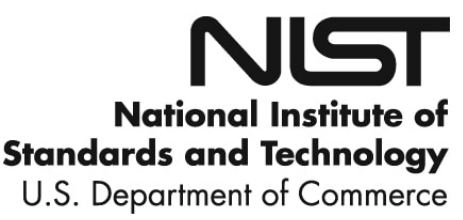


NISTIR 8397

\title{
Guidelines on Minimum Standards for Developer Verification of Software
}

\author{
Paul E. Black \\ Barbara Guttman \\ Vadim Okun \\ Software and Systems Division \\ Information Technology Laboratory
}

This publication is available free of charge from:

https://doi.org/10.6028/NIST.IR.8397

October 2021

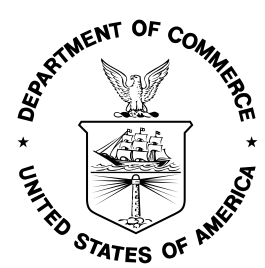

U.S. Department of Commerce Gina M. Raimondo, Secretary

National Institute of Standards and Technology James K. Olthoff, Performing the Non-Exclusive Functions and Duties of the Under Secretary of Commerce for Standards and Technology \& Director, National Institute of Standards and Technology 
Certain commercial entities, equipment, or materials may be identified in this document in order to describe an experimental procedure or concept adequately. Such identification is not intended to imply recommendation or endorsement by the National Institute of Standards and Technology, nor is it intended to imply that the entities, materials, or equipment are necessarily the best available for the purpose.

National Institute of Standards and Technology Interagency or Internal Report 8397

Natl. Inst. Stand. Technol. Interag. Intern. Rep. 8397, 33 pages (October 2021)

This publication is available free of charge from: https://doi.org/10.6028/NIST.IR.8397 


\begin{abstract}
Executive Order (EO) 14028, Improving the Nation's Cybersecurity, 12 May 2021, directs the National Institute of Standards and Technology (NIST) to recommend minimum standards for software testing within 60 days. This document describes eleven recommendations for software verification techniques as well as providing supplemental information about the techniques and references for further information. It recommends the following techniques:

- Threat modeling to look for design-level security issues

- Automated testing for consistency and to minimize human effort

- Static code scanning to look for top bugs

- Heuristic tools to look for possible hardcoded secrets

- Use of built-in checks and protections

- "Black box" test cases

- Code-based structural test cases

- Historical test cases

- Fuzzing

- Web app scanners, if applicable

- Address included code (libraries, packages, services)

The document does not address the totality of software verification, but instead, recommends techniques that are broadly applicable and form the minimum standards.

The document was developed by NIST in consultation with the National Security Agency (NSA). Additionally, we received input from numerous outside organizations through papers submitted to a NIST workshop on the Executive Order held in early June 2021, discussion at the workshop, as well as follow up with several of the submitters.
\end{abstract}

\title{
Keywords
}

software assurance; verification; testing; static analysis; fuzzing; code review; software security.

\section{Additional Information}

For additional information on NIST's Cybersecurity programs, projects, and publications, visit the Computer Security Resource Center. Information on other efforts at NIST and in the Information Technology Laboratory (ITL) is also available.

This document was written at the National Institute of Standards and Technology by employees of the Federal Government in the course of their official duties. Pursuant to Title 17, Section 105 of the United States Code, this is not subject to copyright protection and is in the public domain.

We would appreciate acknowledgment if this document is used. 


\section{Acknowledgments}

The authors particularly thank Fay Saydjari for catalyzing our discussion of scope; Virginia Laurenzano for infusing DevOps Research and Assessments (DORA) principles into the report and other material; Larry Wagoner for numerous contributions and comments; Steve Lipner for reviews and suggestions; David A. Wheeler for extensive corrections and recommendations; and Aurelien M. Delaitre, William Curt Barker, Murugiah Souppaya, Karen Scarfone, and Jim Lyle for their many efforts.

We thank the following for reviewing various codes, standards, guides, and other material: Jessica Fitzgerald-McKay, Hialo Muniz, and Yann Prono. For the acronyms, glossary, and other content, we thank Matthew B. Lanigan, Nhan L. Vo, William C. Totten, and Keith W. Beatty.

We thank all those who submitted position papers applicable for our area to our June 2021 workshop.

This document benefited greatly from additional women and men who shared their insights and expertise during weekly conference calls between NIST and National Security Agency (NSA) staff: Andrew White, Anne West, Brad Martin, Carol A. Lee, Eric Mosher, Frank Taylor, George Huber, Jacob DePriest, Joseph Dotzel, Michaela Bernardo, Philip Scherer, Ryan Martin, Sara Hlavaty, and Sean Weaver. Kevin Stine, NIST, also participated.

We also appreciate contributions from Walter Houser.

\section{Trademark Information}

All registered trademarks or trademarks belong to their respective organizations. 


\section{Table of Contents}

1 Introduction 1

1.1 Overview 1

1.2 Charge 1

1.3 Scope 1

1.4 How Aspects of Verification Relate 3

1.5 Document Outline 4

2 Recommended Minimum Standard for Developer Testing 4

2.1 Threat Modeling 5

2.2 Automated Testing 6

2.3 Code-Based, or Static, Analysis 6

2.4 Review for Hardcoded Secrets 7

2.5 Run with Language-Provided Checks and Protection 7

2.6 Black Box Test Cases

2.7 Code-Based Test Cases 8

2.8 Historical Test Cases $\quad 8$

2.9 Fuzzing 8

2.10 Web Application Scanning $\quad 8$

2.11 Check Included Software Components 9

3 Background and Supplemental Information About Techniques 9

3.1 Supplemental: Built-in Language Protection 9

3.2 Supplemental: Memory-Safe Compilation 10

3.3 Supplemental: Coverage Metrics 11

$\begin{array}{ll}3.4 & \text { Supplemental: Fuzzing } \\ 3.5 & 12\end{array}$

3.5 Supplemental: Web Application Scanning 13

3.6 Supplemental: Static Analysis 14

3.7 Supplemental: Human Reviewing for Properties 15

3.8 Supplemental: Sources of Test Cases 16

3.9 Supplemental: Top Bugs 18

3.10 Supplemental: Checking Included Software for Known Vulnerabilities 18

4 Beyond Software Verification $\quad 19$

4.1 Good Software Development Practices 19

4.2 Good Software Installation and Operation Practices 20

4.3 Additional Software Assurance Technology 21

5 Documents Examined $\quad 22$

6 Glossary and Acronyms 23

$\begin{array}{ll}\text { References } & 23\end{array}$ 


\section{Errata}

In October 2021, we made many grammatical changes due to internal paperwork to obtain a Digital Object Identifier (DOI). While making those, we took the opportunity to also improve or correct text related to Interactive Application Security Testing (IAST) and update the name of an example tool. 


\section{Introduction}

\subsection{Overview}

To ensure that software is sufficiently safe and secure, software must be designed, built, delivered, and maintained well. Frequent and thorough verification by developers as early as possible in the software development life cycle (SDLC) is one critical element of software security assurance. At its highest conceptual level, we may view verification as a mental discipline to increase software quality [1, p. 10]. As NIST's Secure Software Development Framework (SSDF) says, verification is used "to identify vulnerabilities and verify compliance with security requirements" [2, PW.7 and PW.8]. According to International Organization for Standardization (ISO)/ International Electrotechnical Commission (IEC)/ Institute of Electrical and Electronics Engineers (IEEE) 12207:2017 [3, 3.1.72] verification, which is sometimes informally called "testing", encompasses many static and active assurance techniques, tools, and related processes. They must be employed alongside other methods to ensure a high-level of software quality.

This document recommends minimum standards of software verification by software producers. No single software security verification standard can encompass all types of software and be both specific and prescriptive while supporting efficient and effective verification. Thus, this document recommends guidelines for software producers to use in creating their own processes. To be most effective, the process must be very specific and tailored to the software products, technology (e.g., language and platform), toolchain, and development lifecycle model. For information about how verification fits into the larger software development process, see NIST's Secure Software Development Framework (SSDF) [2].

\subsection{Charge}

This document is a response to the 12 May 2021 Executive Order (EO) 14028 on Improving the Nation's Cybersecurity [4]. This document responds to Sec. 4. Enhancing Software Supply Chain Security, subsection (r):

“...guidelines recommending minimum standards for vendors' testing of their software source code, including identifying recommended types of manual or automated testing (such as code review tools, static and dynamic analysis, software composition tools, and penetration testing)." [4, 4(r)]

\subsection{Scope}

This section clarifies or interprets terms that form the basis for the scope of this document.

We define "software" as executable computer programs.

We exclude from our scope ancillary yet vital material such as configuration files, file or execution permissions, operational procedures, and hardware.

Many kinds of software require specialized testing regimes in addition to the minimum standards recommended in Sec. 2. For example, real-time software, firmware (microcode), 
embedded/cyberphysical software, distributed algorithms, machine learning (ML) or neural net code, control systems, mobile applications, safety-critical systems, and cryptographic software. We do not address this specialized testing further. We do suggest minimum testing techniques to use for software that is connected to a network and parallel/multithreaded software.

As a special note, testing requirements for safety-critical systems are addressed by their respective regulatory agencies.

While the EO uses the term "software source code", the intent is much broader and includes software in general including binaries, bytecode, and executables, such as libraries and packages. We acknowledge that it is not possible to examine these as thoroughly and efficiently as human-readable source code.

We exclude from consideration here the verification or validation of security functional requirements and specifications, except as references for testing.

We understand the informal term "testing" as any technique or procedure performed on the software itself to gain assurance that the software will perform as desired, has the necessary properties, and has no important vulnerabilities. We use the ISO/IEC/IEEE term "verification" instead. Verification includes methods such as static analysis and code review, in addition to dynamic analysis or running programs ("testing" in a narrower sense).

We exclude from our treatment of verification other key elements of software development that contribute to software assurance, such as programmer training, expertise, or certification, evidence from prior or subsequent software products, process, correct-byconstruction or model-based methods, supply chain and compilation assurance techniques, and failures reported during operational use.

Verification assumes standard language semantics, correct and robust compilation or interpretation engines, and a reliable and accurate execution environment, such as containers, virtual machines, operating systems, and hardware. Verification may or may not be performed in the intended operational environment.

Note that verification must be based on some references, such as the software specifications, coding standards (e.g., Motor Industry Software Reliability Association (MISRA) $\mathrm{C}$ [5]), collections of properties, security policies, or lists of common weaknesses.

While the EO uses the term "vendors' testing", the intent is much broader and includes developers as well. A developer and a vendor may be the same entity, but many vendors include software from outside sources. A software vendor may redo verification on software packages developed by other entities. Although the EO mentions commercial software [4, Sec. 4(a)], this guideline is written for all software developers, including those employed by the government and developers of open-source software (OSS). The techniques and procedures presented in this document might be used by software developers to verify reused software that they incorporate in their product, customers acquiring software, entities accepting contracted software, or a third-party lab. However, these are not the in- 
tended audience of this document since this assurance effort should be applied as early in the development process as possible.

This document presents "minimum standards". That is, this document is not a guide to most effective practices or recommended practices. Instead, its purposes are to (1) set a lower bar for software verification by indicating techniques that developers should have already been using and (2) serve as a basis for mandated standards in the future.

\subsection{How Aspects of Verification Relate}

This section explains how code-based analysis and reviews relate to dynamic analysis. The fundamental process of dynamic testing of software is shown in Fig. 1. By the time the target software has reached this stage, it should have undergone static analysis by the compiler or other tools. In dynamic testing, the software is run on many test cases, and the outputs are examined. One advantage of dynamic testing is that it has few, if any, false positives. For a general model of dynamic testing see [6, Sec. 3.5.1], which also cites publications.

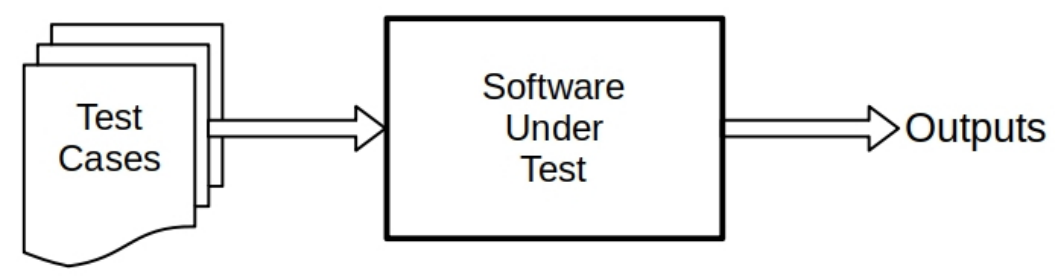

Fig. 1. The basic dynamic testing process is to deliver a set of test cases to the software being tested and examine the outputs.

Verification must be automated in order for thousands of tests to be accurately performed and for the results to be precisely checked. Automation also allows verification to be efficiently repeated often.

Figure 2 provides more details about the process of gaining assurance of software. It shows that some test cases result from a combination of the current set of test cases and from analysis of the code, either entirely by static consideration of the software or by analysis of coverage during test case execution. Sections 2.6 and 2.7 briefly discuss black box and code-based test cases.

Code analysis examines the code itself to check that it has desired properties, to identify weaknesses, and to compute metrics of test completeness. It is also used to diagnose the cause of faults discovered during testing. See Sec. 3.6 for details.

This analysis can determine which statements, routines, paths, etc., were exercised by tests and can produce measures of how complete testing was. Code analysis can also monitor for faults such as exceptions, memory leaks, unencrypted critical information, null pointers, SQL injection, or cross-site scripting. 


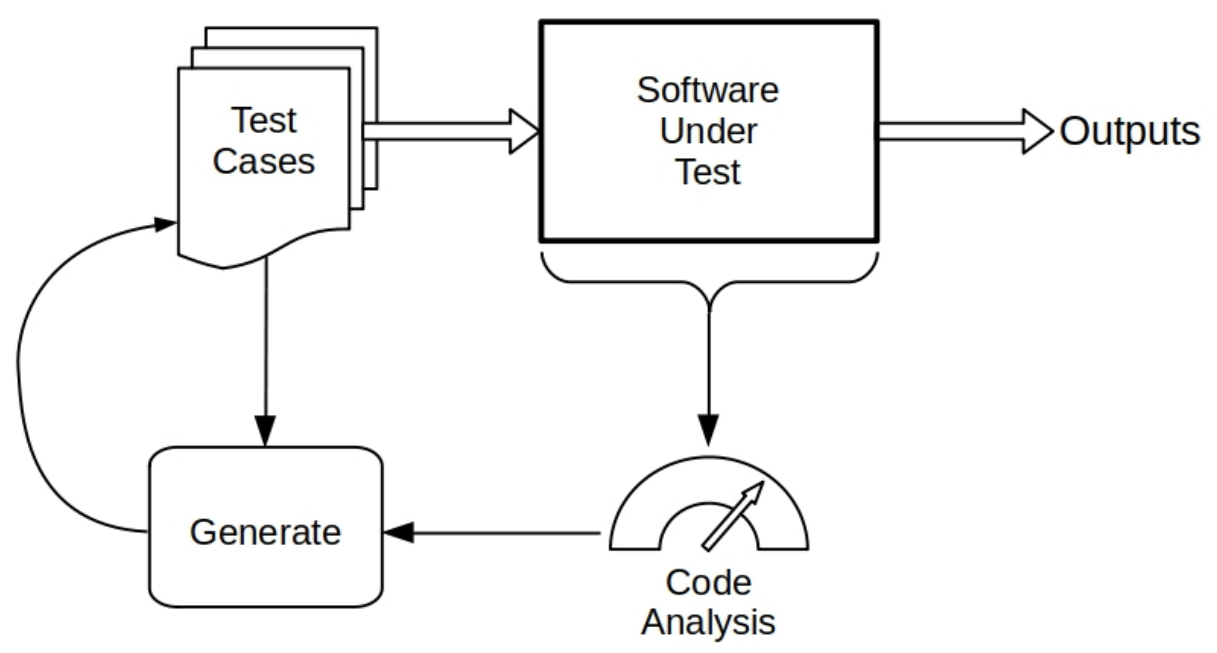

Fig. 2. A more elaborate diagram of the verification process adding how some test cases are generated and showing how code analysis fits.

During testing, such hybrid analysis can also drive active automatic testing, see Secs. 2.9 and 2.10, and is used for Interactive Application Security Testing (IAST). Runtime Application Self-Protection (RASP) monitors the program during operation for internal security faults before they become system failures. IAST and RASP may also scrutinize the output, for example, for sensitive data being transmitted that is not encrypted.

\subsection{Document Outline}

Section 2 begins with a succinct guideline recommending minimum standard techniques for developers to use to verify their software. It then expands on the techniques. Section 3 is informative. That is, it is not part of the recommended minimum. It provides background and supplemental material about the techniques, including references, more thorough variations and alternatives, and example tools. Section 4 summarizes how software must and can be built well from the beginning. Finally, Section 5 lists materials we consulted for this document.

\section{Recommended Minimum Standard for Developer Testing}

Gaining assurance that software does what its developers intended and is sufficiently free from vulnerabilities - either intentionally designed into the software or accidentally inserted at any time during its life cycle-requires the use of many interrelated techniques. This guideline recommends the following minimum standard for developer testing:

- Do threat modeling (see Sec. 2.1).

Using automated testing (Sec. 2.2) for static and dynamic analysis,

- Do static (code-based) analysis

- Use a code scanner to look for top bugs (2.3). 
- Use heuristic tools to look for hardcoded secrets and identify small sections of software that may warrant focused manual code reviews (2.4).

- Do dynamic analysis (i.e., run the program)

- Run the program with built-in checks and protections (2.5).

- Create "black box" test cases, e.g., from specifications, input boundary analysis, and those motivated by threat modeling (2.6).

- Create code-based (structural) test cases. Add cases as necessary to reach at least $80 \%$ coverage (2.7).

- Use test cases that were designed to catch previous bugs (2.8).

- Run a fuzzer (2.9). If the software runs a web service, run a web application scanner, too (2.10).

- Correct the "must fix" bugs that are uncovered and improve the process to prevent similar bugs in the future, or at least catch them earlier [2, RV.3].

- Use similar techniques to gain assurance that included libraries, packages, services, etc., are no less secure than the code (2.11).

The rest of this section provides additional information about each aspect of the recommended minimum standard.

\subsection{Threat Modeling}

We recommend using threat modeling early in order to identify design-level security issues and to focus verification. Threat-modeling methods create an abstraction of the system, profiles of potential attackers, including their goals and methods, and a catalog of potential threats [7]. See also [8]. Shevchenko et al. [7] lists twelve threat-modeling methods, pointing out that software needs should drive the method(s) used. Threat modeling should be done multiple times during development, especially when developing new capabilities, to capture new threats and improve modeling [9]. The DoD Enterprise DevSecOps Reference Design document of August 2019 includes a diagram of how threat modeling fits into software development (Dev), security (Sec), and operations (Ops) [10, Fig. 3]. DevSecOps is an organizational software engineering culture and practice focused on unifying development, security, and operations aspects related to software.

Test cases should be more comprehensive in areas of greatest consequences, as indicated by the threat assessment or threat scenarios. Threat modeling can also indicate which input vectors are of most concern. Testing variations of these particular inputs should be higher priority. Threat modeling may reveal that certain small pieces of code, typically less than 100 lines, pose significant risk. Such code may warrant manual code review to answer specific questions such as, "does the software require authorization when it should?" and "do the software interfaces check and validate input?" See Sec. 3.7 for more on manual reviews. 


\subsection{Automated Testing}

Automated support for verification can be as simple as a script that reruns static analysis, then runs the program on a set of inputs, captures the outputs, and compares the outputs to expected results. It can be as sophisticated as a tool that sets up the environment, runs the test, then checks for success. Some test tools drive the interface to a web-enabled application, letting the tester specify high-level commands, such as "click this button" or "put the following text in a box" instead of maneuvering a mouse pointer to a certain place on a rendered screen and passing events. Advanced tools produce reports of what code passes their tests or summaries of the number of tests passed for modules or subsystems.

We recommend automated verification to

- ensure that static analysis does not report new weaknesses,

- run tests consistently,

- check results accurately, and

- minimize the need for human effort and expertise.

Automated verification can be integrated into the existing workflow or issue tracking system [2, PO.3]. Because verification is automated, it can be repeated often, for instance, upon every commit or before an issue is retired.

\subsection{Code-Based, or Static, Analysis}

Although there are hybrids, analysis may generally be divided into two approaches: 1) code-based or static analysis (e.g., Static Application Security Testing-SAST) and 2) execution-based or dynamic analysis (e.g., Dynamic Application Security Testing-DAST). Pure code-based analysis is independent of program execution. A static code scanner reasons about the code as written, in somewhat the same fashion as a human code reviewer. Questions that a scanner may address include:

- Does this software always satisfy the required security policy?

- Does it satisfy important properties?

- Would any input cause it to fail?

We recommend using a static analysis tool to check code for many kinds of vulnerabilities, see Sec. 3.9, and for compliance with the organization's coding standards. For multi-threaded or parallel processing software, use a scanner capable of detecting race conditions. See Sec. 3.6 for example tools and more guidelines.

Static scanners range in sophistication from simply searching for any use of a deprecated function to looking for patterns indicating possible vulnerabilities to being able to verify that a piece of code faithfully implements a communication protocol. In addition to closed source tools, there are powerful free and open-source tools that provide extensive analyst aids, such as control flows and data values that lead to a violation.

Static source code analysis should be done as soon as code is written. Small pieces of code can be checked before large executable pieces are complete. 


\subsection{Review for Hardcoded Secrets}

We recommend using heuristic tools to examine the code for hardcoded passwords and private encryption keys. Such tools are feasible since functions or services taking these as parameters have specific interfaces. Dynamic testing is unlikely to uncover such unwanted code.

While the primary method to reduce the chance of malicious code is integrity measures, heuristic tools may assist by identifying small sections of code that are suspicious, possibly triggering manual review.

Section 3.7 lists additional properties that might be checked during scans or reviews.

\subsection{Run with Language-Provided Checks and Protection}

Programming languages, both compiled and interpreted, provide many built-in checks and protections. Use such capabilities both during development and in the software shipped [2, PW.6.2]. Enable hardware and operating system security and vulnerability mitigation mechanisms, too (see Sec. 3.1).

For software written in languages that are not memory-safe, consider using techniques that enforce memory safety (see Sec. 3.2).

Interpreted languages typically have significant security enforcement built-in, although additional measures can be enabled. In addition, you may use a static analyzer, sometimes called a "linter", which checks for dangerous functions, problematic parameters, and other possible vulnerabilities (see Sec. 3.6).

Even with these checks, programs must be executed. Executing all possible inputs is impossible except for programs with the tiniest input spaces. Hence, developers must select or construct the test cases to be used. Static code analysis can add assurance in the gaps between test cases, but selective test execution is still required. Many principles can guide the choice of test cases.

\subsection{Black Box Test Cases}

"Black box" tests are not based on the implementation or the particular code. Instead, they are based on functional specifications or requirements, negative tests (invalid inputs and testing what the software should not do) [11, p. 8-5, Sec. 8.B], denial of service and overload, described in Sec. 3.8, input boundary analysis, and input combinations [12, 13].

Tests cases should be more comprehensive in areas indicated as security sensitive or critical by general security principles.

If you can formally prove that classes of errors cannot occur, some of the testing described above may not be needed. Additionally, rigorous process metrics may show that the benefit of some testing is small compared to the cost. 


\subsection{Code-Based Test Cases}

Code-based, or structural, test cases are based on the implementation, that is, the specifics of the code. For instance, suppose the software is required to handle up to one million items. The programmer may decide to implement the software to handle 100 items or fewer in a statically-allocated table but dynamically allocate memory if there are more than 100 items. For this implementation, it is useful to have cases with exactly 99, 100, and 101 items in order to test for bugs in switching between approaches. Memory alignment concerns may indicate additional tests. These important test cases could not have been determined by only considering the specifications.

Code-based test cases may also come from coverage metrics. As hinted at in Fig. 2, when tests are run, the software may record which branches, blocks, function calls, etc., in the code are exercised or "covered". Tools then analyze this information to compute metrics. Additional test cases can be added to increase coverage.

Most code should be executed during unit testing. We recommend that executing the test suite achieves a minimum of $80 \%$ statement coverage [14] (see Sec. 3.3).

\subsection{Historical Test Cases}

Some test cases are created specifically to show the presence (and later, the absence) of a bug. These are sometimes called "regression tests". These test cases are an important source of tests until the process is mature enough to cover them, that is, until a "first principles" assurance approach is adopted that would detect or preclude the bug. An even better option is adoption of an assurance approach, such as choice of language, that precludes the bug entirely.

Inputs recorded from production operations may also be good sources of test cases.

\subsection{Fuzzing}

We recommend using a fuzzer, see Sec. 3.4, which performs automatic active testing; fuzzers create huge numbers of inputs during testing. Typically, only a tiny fraction of the inputs trigger code problems.

In addition, these tools only perform a general check to determine that the software handled the test correctly. Typically, only broad output characteristics and gross behavior, such as application crashes, are monitored.

The advantage of generality is that such tools can try an immense number of inputs with minimal human supervision. The tools can be programmed with inputs that often reveal bugs, such as very long or empty inputs and special characters.

\subsection{Web Application Scanning}

If the software provides a web service, use a dynamic application security testing (DAST) tool, e.g., web application scanner, see Sec. 3.5, or Interactive Application Security Testing (IAST) tool to detect vulnerabilities. 
As with fuzzers, web app scanners create inputs as they run. A web app scanner monitors for general unusual behavior. A hybrid or IAST tool may also monitor program execution for internal faults. When an input causes some detectable anomaly, the tool can use variations of the input to probe for failures.

\subsection{Check Included Software Components}

Use the verification techniques recommended in this section to gain assurance that included code is at least as secure as code developed locally [2, PW.3]. Some assurance may come from self-certification or partially self-certified information, such as the Core Infrastructure Initiative (CII) Best Practices badge [15] or trusted third-party examination.

The components of the software must be continually monitored against databases of known vulnerabilities; a new vulnerability in existing code may be reported at any time.

A Software Composition Analysis (SCA) or Origin Analyzer (OA) tool can help you identify what open-source libraries, suites, packages, bundles, kits, etc., the software uses. These tools can aid in determining what software is really imported, identifying reused software (including open-source software), and noting software that is out of date or has known vulnerabilities (see Sec. 3.10).

\section{Background and Supplemental Information About Techniques}

This section is informative, not part of the recommended minimum. It provides more details about techniques and approaches. Subsections include information such as variations, additional cautions and considerations, example tools, and tables of related standards, guides, or references.

\subsection{Supplemental: Built-in Language Protection}

Programming languages have various protections built into them that preclude some vulnerabilities, warn about poorly written or insecure code, or protect programs during execution. For instance, many languages are memory-safe by default. Others only have flags and options to activate their protections. All such protections should be used as much as possible [2, PW.6.2].

For instance, gcc has flags that enable

- run-time buffer overflow detection,

- run-time bounds checking for $\mathrm{C}++$ strings and containers,

- address space layout randomization (ASLR),

- increased reliability of stack overflow detection,

- stack smashing protector,

- control flow integrity protection,

- rejection of potentially unsafe format string arguments,

- rejection of missing function prototypes, and

- reporting of many other warnings and errors. 
Similarly, the Visual Studio 2019 option “/sdl” enables checks comparable to those described above for gcc.

Interpreted languages typically have significant security enforcement built-in, although additional measures can be enabled. As an example of an interpreted language, Perl has a "taint" mode, enabled by the "-T" command line flag, that "turns on various checks, such as checking path directories to make sure they aren't writable by others." $[16,10.2]$. The "-w" command line option helps as do other measures explained in the Perl security document, perlsec [17]. JavaScript has a "use strict" directive "to indicate that the code should be executed in "strict mode". With strict mode, you cannot, for example, use undeclared variables." [18]

In addition, you may use a static analyzer, sometimes called a "linter", to check for dangerous function or problematic parameters in interpreted languages (see Sec. 3.6).

In addition to capabilities provided by the language itself, you can use hardware (HW) and operating system (OS) mechanisms to ensure control flow integrity, for instance, Intel's Control-flow Enforcement Technology (CET) or ARM Pointer Authentication and landing points. There are compiler options that create opcodes so that if the software is running on hardware, operating systems, or processes with these enabled, these mechanisms will be invoked. All x86 and ARM chips in production have and will have this capability. Most OSs now support it.

Users should also take advantage of HW and OS mechanisms as they update technology by ensuring the HW or OS that they are upgrading to include these HW-based features. These mechanisms help prevent memory corruption bugs that are not detected by verification during development from being exploited.

\begin{tabular}{|l|l|}
\hline Technique, Principle, or Directive & Reference \\
\hline "Applying warning flags" & {$[11$, p. 8-4, Sec. 8.B] } \\
\hline Using stack protection & {$[19]$} \\
\hline Prevent execution of data memory Principle 17 & {$[20$, p. 9] } \\
\hline
\end{tabular}

Table 1. Related Standards, Guides, or References for Built-in Language Protection

\subsection{Supplemental: Memory-Safe Compilation}

Some languages, such as $\mathrm{C}$ and $\mathrm{C}++$, are not memory-safe. A minor memory access error can lead to vulnerabilities such as privilege escalation, denial of service, data corruption, or exfiltration of data.

Many languages are memory-safe by default but have mechanisms to disable those safeties when needed, e.g., for critical performance requirements. Where practical, use memory-safe languages and limit disabling memory safety mechanisms.

For software written languages that are not memory-safe, consider using automated source code transformations or compiler techniques that enforce memory safety.

Requesting memory mapping to a fixed (hardcoded) address subverts address space layout randomization (ASLR). This should be mitigated by enabling appropriate compile 
flag(s) (see Sec. 3.1).

Example Tools

Baggy Bounds Checking, CodeHawk, SoftBoundCETS, and WIT.

\begin{tabular}{|l|l|}
\hline Technique, Principle, or Directive & Reference \\
\hline "Applying warning flags" & {$[11, \mathrm{p} .8-4$, Sec. 8.B] } \\
\hline using stack protection & {$[19]$} \\
\hline $\begin{array}{l}\text { Element A "avoid/detect/remove specific types of vulnera- } \\
\text { bilities at the implementation stage" }\end{array}$ & {$[21, \mathrm{p} .9-12]$} \\
\hline $\begin{array}{l}\text { FPT_AEX_EXT.1 Anti-Exploitation Capabilities "The ap- } \\
\text { plication shall not request to map memory at an explicit ad- } \\
\text { dress except for [assignment: list of explicit exceptions]." }\end{array}$ & {$[22]$} \\
\hline
\end{tabular}

Table 2. Related Standards, Guides, or References for Memory-Safe Compilation

\subsection{Supplemental: Coverage Metrics}

Exhaustive testing is intractable for all but the simplest programs, yet thorough testing is necessary to reduce software vulnerabilities. Coverage criteria are a way to define what needs to be tested and when the testing objective is achieved. For instance, "statement coverage" measures the statements in the code that are executed at least once, i.e., statements that are "covered".

Checking coverage identifies parts of code that have not been thoroughly tested and, thus, are more likely to have bugs. The percentage of coverage, e.g., $80 \%$ statement coverage, is one measure of the thoroughness of a test suite. Test cases can be added to exercise code or paths that were not executed. Low coverage indicates inadequate testing, but very high code coverage guarantees little [14].

Statement coverage is the weakest criterion widely used. For instance, consider an "if" statement with only a "then" branch, that is, without an "else" branch. Knowing that statements in the "then" branch were executed does not guarantee that any test explored what happens when the condition is false and the body is not executed at all. "Branch coverage" requires that every branch is taken. In the absence of early exits, full branch coverage implies full block coverage, so it is stronger than block coverage. Data flow and mutation are stronger coverage criteria [23].

Most generally, “. . . all test coverage criteria can be boiled down to a few dozen criteria on just four mathematical structures: input domains, graphs, logic expressions, and syntax descriptions (grammars)." [1, p. 26, Sec. 2.4] An example of testing based on input domains is combinatorial testing $[12,13]$, which partitions the input space into groups and tests all n-way combinations of the groups. Block, branch, and data flow coverage are graph coverage criteria. Criteria based on logic expressions, such as Modified Condition Decision Coverage (MCDC), require making various truth assignments to the expressions. 
Syntax description criteria are exemplified by mutation testing that deliberately and systematically creates software variants with small syntactic changes that are likely to be errors. For instance, the "less than" operator $(<)$ might be replaced by "greater than or equal to" $(>=)$. If a test set distinguishes the original program from each slight variation, the test set is exercising the program adequately. Mutation testing can be applied to specifications as well as programs.

Note: the code may be compiled with certain flags to measure coverage, then compiled again with different flags for shipment. There needs to be assurance that the source and any included binaries used to build the shipped products match those verified and measured for coverage.

\begin{tabular}{|l|l|}
\hline Technique, Principle, or Directive & Reference \\
\hline "Test coverage analyzer" & {$[11$, p. 8-6, Sec. 8.B] } \\
\hline "Relevant Metrics" & {$[24]$} \\
\hline [ST3.4] "Leverage coverage analysis" & {$[25$, p. 78] } \\
\hline
\end{tabular}

Table 3. Related Standards, Guides, or References for Coverage Metrics

\subsection{Supplemental: Fuzzing}

Fuzzers and related automated randomized test generation techniques are particularly useful when run often during software development. Continuous fuzzing on changing code bases can help catch unexpected bugs early. "Fuzz testing is effective for finding vulnerabilities because most modern programs have extremely large input spaces, while test coverage of that space is comparatively small." [26] Pre-release fuzzing is particularly useful, as it denies malicious parties use of the very same tool to find bugs to exploit.

Fuzzing is a mostly automated process that may require relatively modest ongoing manual labor. It usually requires a harness to feed generated inputs to the software under test. In some case, unit test harnesses may be used. Fuzzing is computationally-intensive and yields best results when performed at scale.

Fuzzing components separately can be efficient and improve code coverage. In this case, the entire system must also be fuzzed as one to investigate whether components work properly when used together.

One key benefit of fuzzing is that it typically produces actual positive tests for bugs, not just static warnings. When a fuzzer finds a failure, the triggering input can be saved and added to the regular test corpus. Developers can use the execution trace leading to the failure to understand and fix the bug. This may not be the case when failures are nondeterministic, for instance, in the presence of threads, multiple interacting processes, or distributed computing.

Fuzzing approaches can be grouped into two categories based on how they create input: mutation based and generation based. Mutation-based fuzzing modifies existing inputs, e.g., from unit tests, to generate new inputs. Generation-based fuzzing produces random inputs from a formal grammar that describes well-formed inputs. Using both gains the 
advantages of both mutation and generation fuzzers. Using both approaches can cover a larger set of test case scenarios, improve code coverage, and increase the chance of finding vulnerabilities missed by techniques such as code reviews.

Mutation-based fuzzing is easy to set up since it needs little or no description of the structure. Mutations to existing inputs may be random or may follow heuristics. Unguided fuzzing typically shallowly explores execution paths. For instance, completely random inputs to date fields are unlikely to be valid. Even most random inputs that are two-digit days (DD), three-letter month abbreviations (Mmm), and four-digit years (YYYY) will be rejected. Constraining days to be 1-31, months to be Jan, Feb, Mar, etc., and years to be within 20 years of today may still not exercise leap-century calculations or deeper logic. Generation-based fuzzing can pass program validation to achieve deeper testing but typically requires far more time and expertise to set up.

Modern mutation-based fuzzers explore execution paths more deeply than unguided fuzzers by using methods such as instrumentation and symbolic execution to take paths that have not yet been explored. Coverage-guided fuzzers, such as AFL++, Honggfuzz, and libFuzzer, aim at maximizing code coverage.

To further improve effectiveness, design review, which should be first done near the beginning of development, may indicate which input vectors are of most concern. Fuzzing these particular inputs should be prioritized.

Fuzzing is often used with special instrumentation to increase the likelihood of detecting faults. For instance, memory issues can be detected by tools such as Address Sanitizer (ASAN) or Valgrind. This instrumentation can cause significant overhead but enables detection of out-of-bounds memory access even if the fault would not cause a crash.

\section{Example Tools}

American Fuzzy Lop Plus Plus (AFL++), Driller, dtls-fuzzer, Eclipser, Honggfuzz, Jazzer, libFuzzer, Mayhem, Peach, Pulsar, Radamsa, and zzuf.

\begin{tabular}{|l|l|}
\hline Technique, Principle, or Directive & Reference \\
\hline $\begin{array}{l}\text { PW.8: Test Executable Code to Identify Vulnerabilities and } \\
\text { Verify Compliance with Security Requirements }\end{array}$ & {$[2]$} \\
\hline "Fuzz testing" & {$[11$, p. 8-5, Sec. 8.B] } \\
\hline Malformed Input Testing (Fuzzing) & {$[27$, slide 8$]$} \\
\hline $\begin{array}{l}\text { [ST2.6] "Perform fuzz testing customized to application } \\
\text { API" }\end{array}$ & {$[25$, p. 78$]$} \\
\hline
\end{tabular}

Table 4. Related Standards, Guides, or References for Fuzzing

\subsection{Supplemental: Web Application Scanning}

DAST tools, such as web app scanners, test software in operation. IAST tools check software in operation. Such tools may be integrated with user interface (UI) and rendering packages so that the software receives button click events, selections, and text submissions 
in fields, exactly as it would in operation. These tools then monitor for subtle hints of problems, such as an internal table name in an error message. Many web app scanners include fuzzers.

Internet and web protocols require a huge amount of complex processing that have historically been a source of serious vulnerabilities.

Penetration testing is a "test methodology in which assessors, typically working under specific constraints, attempt to circumvent or defeat the security features of an information system." [28] That is, it is humans using tools, technologies, and their knowledge and expertise to simulate attackers in order to detect vulnerabilities and exploits.

\section{Example Tools}

Acunetix, AppScan, AppSpider, Arachni, Burp, Contrast, Grabber, IKare, Nessus, Probely, SQLMap, Skipfish, StackHawk, Vega, Veracode DAST, W3af, Wapiti, WebScarab, Wfuzz, and Zed Attack Proxy (ZAP).

\begin{tabular}{|l|l|}
\hline Technique, Principle, or Directive & Reference \\
\hline "Web application scanner" & {$[11, \mathrm{p} .8-5, \mathrm{Sec} .8 . \mathrm{B}]$} \\
\hline $\begin{array}{l}\text { "Security Testing in the Test/Coding Phase", subsection } \\
\text { "System Testing" }\end{array}$ & {$[24]$} \\
\hline $\begin{array}{l}\text { [ST2.1] "Integrate black-box security tools into the QA } \\
\text { process" }\end{array}$ & {$[25, \mathrm{p} .77]$} \\
\hline $\begin{array}{l}\text { 3.12.1e "Conduct penetration testing [Assignment: } \\
\text { organization-defined frequency], leveraging automated } \\
\text { scanning tools and ad hoc tests using subject matter } \\
\text { experts." }\end{array}$ & \\
\hline
\end{tabular}

Table 5. Related Standards, Guides, or References for Web Application Scanning

\subsection{Supplemental: Static Analysis}

Static analysis or Static Application Security Testing (SAST) tools, sometimes called "scanners", examine the code, either source or binary, to warn of possible weaknesses. Use of these tools enable early, automated problem detection. Some tools can be accessed from within an Integrated Development Environment (IDE), providing developers with immediate feedback. Scanners can find issues such as buffer overflows, SQL injections, and noncompliance with an organization's coding standards. The results may highlight the precise files, line numbers, and even execution paths that are affected to aid correction by developers.

Organizations should select and standardize on static analysis tools and establish lists of "must fix" bugs based on their experience with the tool, the applications under development, and reported vulnerabilities. You may consult published lists of top bugs (see Sec. 3.9) to help create a process-specific list of "must fix" bugs. 
SAST scales well as tests can be run on large software and can be run repeatedly, as with nightly builds for the whole system or in developers' IDE.

SAST tools have weaknesses, too. Certain types of vulnerabilities are difficult to find, such as authentication problems, access control issues, and insecure use of cryptography. In almost all tools, some warnings are false positives, and some are insignificant in the context of the software. Further, tools usually cannot determine if a weakness is an actual vulnerability or is mitigated in the application. Tool users should apply warning suppression and prioritization mechanisms provided by tools to triage the tool results and focus their effort on correcting the most important weaknesses.

Scanners have different strengths because of code styles, heuristics, and relative importance classes of vulnerabilities have to the process. You can realize the maximum benefit by running more than one analyzer and paying attention to the weakness classes for which each scanner is best.

Many analyzers allow users to write rules or patterns to increase the analyzer's benefit.

\section{Example Tools}

Astrée, Polyspace Bug Finder, Parasoft C/C++test, Checkmarx SAST, CodeSonar, Coverity, Fortify, Frama-C, Klocwork, SonarSource, SonarQube, and Veracode SAST handle many common compiled languages.

For JavaScript, JSLint, JSHint, PMD, and ESLint.

\begin{tabular}{|l|l|}
\hline Technique, Principle, or Directive & Reference \\
\hline $\begin{array}{l}\text { PW.8: Test Executable Code to Identify Vulnerabilities and } \\
\text { Verify Compliance with Security Requirements }\end{array}$ & {$[2]$} \\
\hline $\begin{array}{l}\text { "Source code quality analyzers" } \\
\text { "Source code weakness analyzers" }\end{array}$ & {$[11, \mathrm{p} .8-5$, Sec. $8 . B]$} \\
\hline $\begin{array}{l}\text { [CR1.4] "Use automated tools along with manual review" } \\
\text { [CR2.6] "Use automated tools with tailored rules" }\end{array}$ & {$[25$, pp. $75-76]$} \\
\hline
\end{tabular}

Table 6. Related Standards, Guides, or References for Static Analysis

\subsection{Supplemental: Human Reviewing for Properties}

As discussed in Sec. 3.6, static analysis tools scan for many properties and potential problems. Some properties are poorly suited to computerized recognition, and hence may warrant human examination. This examination may be more efficient with scans that indicate possible problems or locations of interest.

Someone other than the original author of the code may review it to ensure that it

- performs bounds checks [19],

- sets initial values for data [19],

- only allows authorized users to access sensitive transactions, functions, and data [30, p. 10, Sec. 3.1.2] (may include check that user functionality is separate from system management functionality [30, p. 37, Sec. 3.13.3]), 
- limits unsuccessful logon attempts [30, p. 12, Sec. 3.1.8],

- locks a session after period of inactivity [30, p. 13, Sec. 3.1.10],

- automatically terminates session after defined conditions [30, p. 13, Sec. 3.1.11],

- has an architecture that "promote[s] effective information security within organizational systems" [30, p. 36, Sec. 3.13.2],

- does not map memory to hardcoded locations, see Sec. 3.2,

- encrypts sensitive data for transmission [30, p. 14, Sec. 3.1.13] and storage [30, p. 15, Sec. 3.1.19].

- uses standard services and application program interfaces (APIs) [2, PW.4],

- has a secure default configuration [2, PW.9], and

- has an up-to-date documented interface.

A documented interface includes the inputs, options, and configuration files. The interface should be small, to reduce the attack surface [31, p. 15].

Threat modeling may indicate that certain code poses significant risks. A focused manual review of small pieces, typically less than 100 lines, of code may be beneficial for the cost. The review could answer specific questions. For example, does the software require authorization when it should? Do the software interfaces check and validate inputs?

\begin{tabular}{|l|l|}
\hline Technique, Principle, or Directive & Reference \\
\hline "Focused manual spot check" & {$[11, \mathrm{p} .5-6$, Sec. 5.A] } \\
\hline $\begin{array}{l}\text { 3.14.7e "Verify the correctness of [Assignment: } \\
\text { organization-defined security critical or essential software, }\end{array}$ & {$[29]$} \\
$\begin{array}{l}\text { firmware, and hardware components] using [Assignment: } \\
\text { organization-defined verification methods or techniques] }\end{array}$ & \\
\hline
\end{tabular}

Table 7. Related Standards, Guides, or References for Human Reviewing for Properties

\subsection{Supplemental: Sources of Test Cases}

Typically, tests are based on specifications or requirements, which are designed to ensure the software does what it is supposed do, and process experience, which is designed to ensure previous bugs do not reappear. Additional test cases may be based on the following principles:

- Threat modeling-concentrate on areas with highest consequences,

- General security principles-find security vulnerabilities, such as failure to check credentials, since these often do not cause operational failures (crashes or incorrect output),

- Negative tests-make sure that software behaves reasonably for invalid inputs and that it does not do what it should not do, for instance ensure that a user cannot perform operations for which they are not authorized [11, p. 8-5, Sec. 8.B],

- Combinatorial testing-find errors occurring when handling certain n-tuples of kinds of input [12, 13], and

- Denial of service and overload—make sure software is resilient. 
Denial of service and overload tests are also called stress testing. Also consider algorithmic attacks. Algorithms may work well with a typical load or expected overload, but an attacker may cause a load many orders of magnitude higher than would ever occur in actual use.

Monitor execution and output during negative testing especially, such as with Interactive Application Security Testing (IAST) tools or Runtime Application Self-Protection (RASP).

\begin{tabular}{|c|c|}
\hline Technique, Principle, or Directive & Reference \\
\hline $\begin{array}{l}\text { "Simple attack modeling" } \\
\text { "Negative testing" }\end{array}$ & $\begin{array}{l}{[11, \text { pp. } 8-4 \text { and } 8-5 \text {, }} \\
\text { Sec. } 8 . B]\end{array}$ \\
\hline $\begin{array}{l}\text { "Security Testing in the Test/Coding Phase", subsection } \\
\text { "Unit Testing" and subsection "System Testing" } \\
\text { "Security Testing Activities", subsection "Risk Analysis" }\end{array}$ & $e_{1}$ \\
\hline $\begin{array}{l}\text { [AM1.2] "Create a data classification scheme and inven- } \\
\text { tory" } \\
\text { [AM1.3] "Identify potential attackers" } \\
\text { [AM2.1] "Build attack patterns and abuse cases tied to po- } \\
\text { tential attackers" } \\
\text { [AM2.2] "Create technology-specific attack patterns" } \\
\text { [AM2.5] "Build and maintain a top N possible attacks list" } \\
\text { [AM3.2] "Create and use automation to mimic attackers" }\end{array}$ & {$[25$, pp. 67-68] } \\
\hline $\begin{array}{l}\text { [ST1.1] "Ensure QA performs edge/boundary value condi- } \\
\text { tion testing" } \\
\text { [ST1.3] "Drive tests with security requirements and secu- } \\
\text { rity features" } \\
\text { [ST3.3] "Drive tests with risk analysis results" }\end{array}$ & {$[25$, pp. 77-78] } \\
\hline $\begin{array}{l}\text { [SE1.1] "Use application input monitoring" } \\
\text { [SE3.3] "Use application behavior monitoring and diagnos- } \\
\text { tics" }\end{array}$ & {$[25, \mathrm{pp} .80$ and 82$]$} \\
\hline $\begin{array}{l}\text { 3.11.1e Employ [Assignment: organization-defined } \\
\text { sources of threat intelligence] as part of a risk assessment } \\
\text { to guide and inform the development of organizational } \\
\text { systems, security architectures, selection of security } \\
\text { solutions, monitoring, threat hunting, and response and } \\
\text { recovery activities } \\
\text { 3.11.4e Document or reference in the system security plan } \\
\text { the security solution selected, the rationale for the security } \\
\text { solution, and the risk determination }\end{array}$ & [29] \\
\hline
\end{tabular}

Table 8. Related Standards, Guides, or References for Sources of Test Cases 


\subsection{Supplemental: Top Bugs}

There are many collections of high priority bugs and weaknesses, such as those identified in the Common Weakness Enumeration (CWE)/SANS Top 25 Most Dangerous Software Errors [32, 33], the CWE Weaknesses on the Cusp [34], or the Open Web Application Security Project (OWASP) Top 10 Web Application Security Risks [35].

These lists, along with experience with bugs found, can help developers begin choosing bug classes to focus on during verification and process improvement.

\begin{tabular}{|l|l|}
\hline Technique, Principle, or Directive & Reference \\
\hline "UL and Cybersecurity" & {$[27$, slide 8] } \\
\hline $\begin{array}{l}\text { For security "Code Quality Rules" lists 36 “parent" CWEs } \\
\text { and } 38 \text { "child" CWEs. For reliability, it lists 35 "parent" }\end{array}$ \\
CWEs and 39 "child" CWEs. & \\
\hline
\end{tabular}

Table 9. Related Standards, Guides, or References for Top Bugs

\subsection{Supplemental: Checking Included Software for Known Vulnerabilities}

You need to have as much assurance for included code, e.g., closed source software, free and open-source software, libraries, and packages, as for code you develop. If you lack strong guarantees, we recommend as much testing of included code as of the code.

Earlier versions of packages and libraries may have known vulnerabilities that are corrected in later versions.

Software Composition Analysis (SCA) and Origin Analyzer (OA) tools scan a code base to identify what code is included. They also check for any vulnerabilities that have been reported for the included code [11, App. C.21, p. C-44]. A widely-used database of publicly known vulnerabilities is the NIST National Vulnerability Database (NVD), which identifies vulnerabilities using Common Vulnerabilities and Exposures (CVE). Some tools can be configured to prevent download of software that has security issues and recommend alternative downloads.

Since libraries are matched against a tool's database, they will not identify libraries missing from the database.

\section{Example Tools}

Black Duck, Binary Analysis Tool (BAT), Contrast Assess, FlexNet Code Insight, FOSSA, JFrog Xray, OWASP Dependency-Check, Snyk, Sonatype IQ Server, Veracode SCA, WhiteHat Sentinel SCA, and WhiteSource Bolt. 


\begin{tabular}{|l|l|}
\hline Technique, Principle, or Directive & Reference \\
\hline "Origin Analyzer" & $\begin{array}{l}{[11, \text { App. C.21, p. C- }} \\
44]\end{array}$ \\
\hline "UL and Cybersecurity" & {$[27$, slide 8$]$} \\
\hline $\begin{array}{l}\text { 3.4.3e Employ automated discovery and management tools } \\
\text { to maintain an up-to-date, complete, accurate, and readily } \\
\text { available inventory of system components }\end{array}$ & {$[29]$} \\
\hline
\end{tabular}

Table 10. Related Standards, Guides, or References for Checking Included Software for Known Vulnerabilities

\section{Beyond Software Verification}

Good software must be built well from the beginning. Verification is just one element in delivering software that meets operational security requirements. The software assurance techniques listed above are just the minimum steps to use in improving the security of enterprise supply chains. Section 4.1 describes a few general software development practices and how assurance fits into the larger subject of secure software development and operation. Even software that has solid security characteristics can be exploited by adversaries if its installation, operation, or maintenance is conducted in a manner that introduces vulnerabilities. Section 4.2 describes some trends and technologies that may improve software assurance. Section 4.3 describes good installation and operation principles. Both software development and security technologies are constantly evolving.

\subsection{Good Software Development Practices}

Ideally, software is secure by design, and the security of both the design and its implementation can be demonstrated, documented, and maintained. Software development, and indeed the full software development lifecycle, has changed over time, but some basic principles apply in all cases. NIST developed a cybersecurity white paper, "Mitigating the Risk of Software Vulnerabilities by Adopting a Secure Software Development Framework (SSDF)" [2], that provides an overview and references about these basic principles. This document is part of an ongoing project; see https://csrc.nist.gov/Projects/ssdf. The SSDF introduces a software development framework of fundamental, sound, and secure software development practices based on established secure software development practice documents. For verification to be most effective, it should be a part of the larger software development process. SSDF practices are organized into four groups:

- Prepare the Organization (PO): Ensure that the organization's people, processes, and technology are prepared at the organization level and, in some cases, for each individual project to develop secure software.

- Protect the Software (PS): Protect all components of the software from tampering and unauthorized access. 
- Produce Well-Secured Software (PW): Produce well-secured software that has minimal security vulnerabilities in its releases.

- Respond to Vulnerabilities (RV): Identify vulnerabilities in software releases, respond appropriately to address those vulnerabilities, and prevent similar vulnerabilities from occurring in the future.

In the context of DevOps, enterprises with secure development include the following characteristics:

- The enterprise creates a culture where security is everyone's responsibility. This includes integrating a security specialist into the development team, training all developers to know how to design and implement secure software, and using automated tools that allow both developers and security staff to track vulnerabilities.

- The enterprise uses tools to automate security checking, often referred to as Security as Code [38].

- The enterprise tracks threats and vulnerabilities, in addition to typical system metrics.

- The enterprise shares software development task information, security threat, and vulnerability knowledge between the security team, developers, and operations personnel.

\subsection{Good Software Installation and Operation Practices}

As stated above, even software that has no identified security vulnerabilities can be subject to exploitation by adversaries if its installation, operation, or maintenance introduces vulnerabilities. Some issues that are not directly addressed in this paper include misconfiguration, violation of file permission policies, network configuration violations, and acceptance of counterfeit or altered software. See especially "Security Measures for "EO-Critical Software" Use Under Executive Order (EO) 14028”, https://www.nist.gov/itl/executive-orderimproving-nations-cybersecurity/security-measures-eo-critical-software-use-under, which addresses patch management, configuration management, and continuous monitoring, among other security measures, and also provides a list of references.

Configuration files: Because of the differences in software applications and networking environments, the parameters and initial settings for many computer applications, server processes, and operating systems are configurable. Often, security verification fails to anticipate unexpected settings. Systems and network operators often alter settings to facilitate tasks that are more difficult or infeasible when using restrictive settings. Particularly in cases of access authorization and network interfaces, changing configuration settings can introduce critical vulnerabilities. Software releases should include secure default settings and caveats regarding deviations from those settings. Security verification should include all valid settings and (possibly) assurance that invalid settings will be caught by run-time checks. The acquirer should be warned or notified that settings other than those explicitly permitted will invalidate developer's security assertions.

File Permissions: File ownership and permissions to read, write, execute, and delete files need to be established using the principle of least privilege. No matter how thoroughly 
software has been verified, security is compromised if it can be modified or if files can be accessed by unauthorized entities. The ability to change file permissions needs to be restricted to explicitly authorized subjects that are authenticated in a manner that is commensurate with the impact of a compromise of the software. The role of file permissions in maintaining security assertions needs to be explicit.

Network configuration: Secure configuration refers to security measures that are implemented when building and installing computers and network devices to reduce cyber vulnerabilities. Just as file permissions are critical to the continued integrity of software, so does network configuration constrain unauthorized access to software. Verification needs to cover all valid network configuration settings and (possibly) provide assurance that invalid settings will be caught by run-time checks. The role of network configuration in scoping the applicability of security assertions needs to be explicit.

Operational configuration: Software is employed in a context of use. Addition or deletion of components that are dependent on a software product or on which the product depends can either validate or invalidate the assumptions on which the security of software and system operation depend. Particularly in the case of source code, the operating code itself depends on components such as compilers and interpreters. In such cases, the security of the software can be invalidated by the other products. Verification needs to be conducted in an environment that is consistent with the anticipated operational configurations. Any dependence of the security assertions on implementing software or other aspects of operational configuration needs to be made explicit by the developer. Supply chain integrity must be maintained.

\subsection{Additional Software Assurance Technology}

Software verification continues to improve as new methods are developed and existing methods are adapted to changing development and operational environments. Some challenges remain, e.g., applying formal methods to prove the correctness of poorly designed code. Nearer-term advances that may add to security assurance based on verification include:

- Applying machine learning to reduce false positives from automated security scanning tools and to increase the vulnerabilities that these tools can detect.

- Adapting tools designed for automated web interface tests, e.g., Selenium, to produce security tests for applications.

- Improving scalability of model-based security testing for complex systems.

- Improving automated web-application security assessment tools with respect to:

- Session state management

- Script parsing

- Logical flow

- Custom uniform resource locators (URLs)

- Privilege escalation

- Applying observability tools to provide security assurance in cloud environments. 
- Adapting current security testing to achieve cloud service security assurance.

Other techniques to reduce software vulnerabilities are described in "Dramatically Reducing Software Vulnerabilities", NIST-IR 8151 [39].

\section{Documents Examined}

This section lists some of the standards, guides, references, etc., that we examined to assemble this document. We list them to give future work an idea where to start or quickly learn what may have been overlooked. We group related references.

Donna Dodson, Murugiah Souppaya, and Karen Scarfone, "Mitigating the Risk of Software Vulnerabilities by Adopting a Secure Software Development Framework (SSDF)", 2013 [2].

David A. Wheeler and Amy E. Henninger, "State-of-the-Art Resources (SOAR) for Software Vulnerability Detection, Test, and Evaluation 2016", 2016 [11].

Steven Lavenhar, "Code Analysis", 2008 [19].

C.C. Michael, Ken van Wyk, and Will Radosevich, "Risk-Based and Functional Security Testing", 2013 [24].

UL, "IoT Security Top 20 Design Principles”, 2017 [20].

Tom Haigh and Carl E. Landwehr, "Building Code for Medical Device Software Security", 2015 [21].

Ulf Lindqvist and Michael Locasto, "Building Code for the Internet of Things", 2017 [31].

Carl E. Landwehr and Alfonso Valdes, "Building Code for Power System Software Security", 2017 [40].

“Protection Profile for Application Software Version 1.3", 2019 [22].

Ron Ross, Victoria Pillitteri, Gary Guissanie, Ryan Wagner, Richard Graubart, and Deb Bodeau, "Enhanced Security Requirements for Protecting Controlled Unclassified Information: A Supplement to NIST Special Publication 800-171”, 2021 [29].

Ron Ross, Victoria Pillitteri, Kelley Dempsey, Mark Riddle, and Gary Guissanie, "Protecting Controlled Unclassified Information in Nonfederal Systems and Organizations", 2020 [30].

Ron Ross, Victoria Pillitteri, and Kelley Dempsey, "Assessing Enhanced Security Requirements for Controlled Unclassified Information”, 2021 [41].

Bill Curtis, Bill Dickenson, and Chris Kinsey, "CISQ Recommendation Guide: Effective Software Quality Metrics for ADM Service Level Agreements”, 2015 [42].

“Coding Quality Rules”, 2021 [36]. 


\section{Glossary and Acronyms}

\section{Term}

Cybersecurity

Software Source Code

\section{Definition}

The practice of protecting systems, networks, and programs from digital attacks.

The software as it is originally entered in plain text, e.g., human-readable alphanumeric characters.
API Application Program Interface
CVE Common Vulnerabilities and Exposures
CWE Common Weakness Enumeration
DAST Dynamic Application Security Testing
EO Executive Order
HW Hardware
IAST Interactive Application Security Testing
MISRA Motor Industry Software Reliability Association
NIST National Institute of Standards and Technology
NSA National Security Agency
NVD National Vulnerability Database
OA Origin Analyzer
OS Operating System
OSS Open Source Software
OWASP Open Web Application Security Project
RASP Runtime Application Self-Protection
SAST Static Application Security Testing
SCA Software Composition Analysis
SDLC Software Development Life Cycle
SSDF Secure Software Development Framework
URL Uniform Resource Locator

\section{References}

[1] Ammann P, Offutt J (2017) Introduction to Software Testing (Cambridge University Press), 2nd Ed. https://doi.org/10.1017/9781316771273

[2] Dodson D, Souppaya M, Scarfone K (2013) Mitigating the risk of software vulnerabilities by adopting a secure software development framework (SSDF) (National Institute of Standards and Technology, Gaithersburg, MD), Cybersecurity White Paper. https://doi.org/10.6028/NIST.CSWP.04232020

[3] (2017) ISO/IEC/IEEE 12207: Systems and software engineering—software life cycle processes (ISO/IEC/IEEE), First edition. 
[4] Biden JR Jr (2021) Improving the nation's cybersecurity, https://www.federalregister. gov/d/2021-10460. Executive Order 14028, 86 FR 26633, Document number 202110460, Accessed 17 May 2021.

[5] Motor Industry Software Reliability Association (2013) MISRA C:2012: Guidelines for the Use of the C Language in Critical Systems (MIRA Ltd, Nuneaton, Warwickshire CV10 0TU, UK).

[6] Black PE (2019) Formal methods for statistical software (National Institute of Standards and Technology, Gaithersburg, MD), IR 8274. https://doi.org/10.6028/NIST.IR. 8274

[7] Shevchenko N, Chick TA, O'Riordan P, Scanlon TP, Woody C (2018) Threat modeling: A summary of available methods, https://resources.sei.cmu.edu/asset files/ WhitePaper/2018_019_001_524597.pdf. Accessed 8 June 2021.

[8] Shostack A (2014) Threat Modeling: Designing for Security (John Wiley \& Sons, Inc.).

[9] Keane J (2021), personal communication.

[10] Chief Information Officer (2019) DoD enterprise DevSecOps reference design (Department of Defense), Version 1.0. Accessed 8 June 2021. Available at https://dodcio. defense.gov/Portals/0/Documents/DoD Enterprise DevSecOps Reference Design v1. 0_Public Release.pdf.

[11] Wheeler DA, Henninger AE (2016) State-of-the-art resources (SOAR) for software vulnerability detection, test, and evaluation 2016 (Institute for Defense Analyses), P-8005. Accessed 19 May 2021. Available at https://www.ida.org/researchand-publications/publications/all/s/st/stateoftheart-resources-soar-for-softwarevulnerability-detection-test-and-evaluation-2016.

[12] Kuhn R, Kacker R, Lei Y, Hunter J (2009) Combinatorial software testing. Computer 42(8):94-96. https://doi.org/10.1109/MC.2009.253. Available at https://tsapps. nist.gov/publication/get_pdf.cfm?pub_id=903128

[13] Kuhn DR, Bryce R, Duan F, Ghandehari LS, Lei Y, Kacker RN (2015) Combinatorial Testing: Theory and Practice (Elsevier), Advances in Computers, Vol. 99, Chapter 1, pp 1-66. https://doi.org/10.1016/bs.adcom.2015.05.003

[14] Cornett S (2013) Minimum acceptable code coverage, https://www.bullseye.com/ minimum.html. Accessed 5 July 2021.

[15] (2018) CII best practices badge program, https://bestpractices.coreinfrastructure.org/. Accessed 25 June 2021.

[16] Wheeler DA (2015) Secure programming HOWTO, https://dwheeler.com/secureprograms/Secure-Programs-HOWTO/. Accessed 24 June 2021.

[17] (2013) perlsec, https://www.linux.org/docs/man1/perlsec.html. Accessed 24 June 2021.

[18] (2021) JavaScript use strict, https://www.w3schools.com/js/js_strict.asp. Accessed 25 June 2021.

[19] Lavenhar S (2008) Code analysis, https://us-cert.cisa.gov/bsi/articles/best-practices/ code-analysis/code-analysis. Accessed 5 May 2021. 
[20] UL (2017) IoT security top 20 design principles, https://ims.ul.com/sites/ g/files/qbfpbp196/files/2018-05/iot-security-top-20-design-principles.pdf. Accessed 12 May 2021.

[21] Haigh T, Landwehr CE (2015) Building code for medical device software security, https://ieeecs-media.computer.org/media/technical-activities/CYBSI/docs/ BCMDSS.pdf. Accessed 15 April 2021.

[22] (2019) Protection profile for application software version 1.3, https://www.niapccevs.org/MMO/PP/PP_APP_v1.3.pdf. Accessed 27 April 2021.

[23] Zhu H, Hall PAV, May JHR (1997) Software unit test coverage and adequacy. ACM Computing Surveys 29(4):366-427. https://doi.org/10.1145/267580.267590

[24] Michael C, van Wyk K, Radosevich W (2013) Risk-based and functional security testing, https://us-cert.cisa.gov/bsi/articles/best-practices/security-testing/risk-basedand-functional-security-testing. Accessed 4 May 2021.

[25] Migues S, Steven J, Ware M (2020) Building security in maturity model (BSIMM) version 11 (Synopsys), Downloaded 3 April 2021. Available at https://www.bsimm. com/download.html.

[26] Okun V, Fong E (2015) Fuzz testing for software assurance. Crosstalk, The Journal of Defense Software Engineering 28:35-37.

[27] UL (2011) UL and cybersecurity, https://alamembers.com/Portals/1/ 10_3 Joseph UL and Cybersecurity.pdf. Accessed 12 May 2021.

[28] (2015) CNSSI 4009 Committee on National Security Systems (CNSS) glossary, $\quad$ https://www.serdp-estcp.org/Tools-and-Training/Installation-Energy-andWater/Cybersecurity/Resources-Tools-and-Publications/Resources-and-ToolsFiles/CNSSI-4009-Committee-on-National-Security-Systems-CNSS-Glossary. Accessed 8 July 2021.

[29] Ross R, Pillitteri V, Guissanie G, Wagner R, Graubart R, Bodeau D (2021) Enhanced security requirements for protecting controlled unclassified information: A supplement to NIST special publication 800-171 (National Institute of Standards and Technology, Gaithersburg, MD), SP 800-172. https://doi.org/10.6028/NIST.SP.800-172

[30] Ross R, Pillitteri V, Dempsey K, Riddle M, Guissanie G (2020) Protecting controlled unclassified information in nonfederal systems and organizations (National Institute of Standards and Technology, Gaithersburg, MD), SP 800-171r2. https://doi.org/10. 6028/NIST.SP.800-171r2

[31] Lindqvist U, Locasto M (2017) Building code for the internet of things, https://ieeecs-media.computer.org/media/technical-activities/CYBSI/docs/ Building_Code_IoT_online.pdf. Accessed 28 April 2021.

[32] (2021) About CWE, https://cwe.mitre.org/about/. Accessed 25 May 2021.

[33] (2021) CWE/SANS top 25 most dangerous software errors, https://www.sans.org/ top25-software-errors/. Accessed 26 May 2021.

[34] (2020) 2020 CWE top 25 most dangerous software weaknesses, https://cwe.mitre.org/ top25/. Accessed 26 May 2021.

[35] (2020) OWASP top ten, https://owasp.org/www-project-top-ten/. Accessed 26 May 
2021.

[36] Consortium for Information \& Software Quality (2021) Coding quality rules, https: //www.it-cisq.org/coding-rules/. Accessed 13 May 2021.

[37] (2019) List of weaknesses included in the CISQ automated source code quality measures (Consortium for Information \& Software Quality), Accessed 13 May 2021.

[38] Boyer J (2018) Security as code: Why a mental shift is necessary for secure DevOps, https://simpleprogrammer.com/security-code-secure-devops/. Accessed 10 June 2021.

[39] Black PE, Badger L, Guttman B, Fong E (2016) Dramatically reducing software vulnerabilities: Report to the White House Office of Science and Technology Policy (National Institute of Standards and Technology), NISTIR 8151. https://doi.org/ 10.6028/NIST.IR.8151

[40] Landwehr CE, Valdes A (2017) Building code for power system software security, https://ieeecs-media.computer.org/media/technical-activities/CYBSI/docs/ BCPSSS.pdf. Accessed 28 April 2021.

[41] Ross R, Pillitteri V, Dempsey K (2021) Assessing enhanced security requirements for controlled unclassified information (National Institute of Standards and Technology, Gaithersburg, MD), SP 800-172A (Draft). https://doi.org/10.6028/NIST.SP.800172A-draft. Accessed 21 May 2021.

[42] Curtis B, Dickenson B, Kinsey C (2015) CISQ recommendation guide: Effective software quality metrics for ADM service level agreements (Consortium for Information \& Software Quality), Accessed 13 May 2021. 